\title{
Improving uptake of HIV testing services and linkage to care after diagnosis in Kenya
}

Project SOAR

Follow this and additional works at: https://knowledgecommons.popcouncil.org/departments_sbsr-hiv

Part of the Demography, Population, and Ecology Commons, Family, Life Course, and Society Commons, International Public Health Commons, and the Medicine and Health Commons How does access to this work benefit you? Let us know!

\section{Recommended Citation}

Project SOAR. 2017. "Improving uptake of HIV testing services and linkage to care after diagnosis in Kenya," Activity brief. Washington, DC: Population Council. 


\section{Improving Uptake of HIV Testing Services and Linkage to Care after Diagnosis in Kenya}

HIV testing services (HTS) are an important component of HIV prevention in Kenya and provide an entry point into clinical care for persons newly diagnosed with HIV. Although uptake of HIV testing has increased in Kenya, men are less likely than women to get tested. In Nairobi, for example, less than half as many men as women had undergone HIV testing in the 12 months prior to the 2014 Kenya Demographic and Health Survey. ${ }^{1}$ Understanding how and why certain men elect to be tested could provide important insights in designing strategies to improve uptake and expansion of HTS among males.

Another challenge in Kenya is how to identify and address the particular health system, social, and individual factors that hinder each patient's linkage to and retention in care. A tool that assesses these factors and guides health workers in directing HIV-positive clients-both males and females-to particular post-test actions or services based on their specific needs may ensure more optimal service utilization.

Project SOAR is responding to these issues by conducting two complementary research activities. The objective of the first activity is to understand the barriers to and facilitators of HIV testing among men seeking HTS, and the second aims to develop and pilot test an HIV post-test assessment tool to

Research Partners: Population Council, Kenya National AIDS \& STI Control Program

Location: Nairobi County

Study Duration: 2016-2018

For more information, contact Jerry Okal, Population Council, jokal@popcouncil.org.

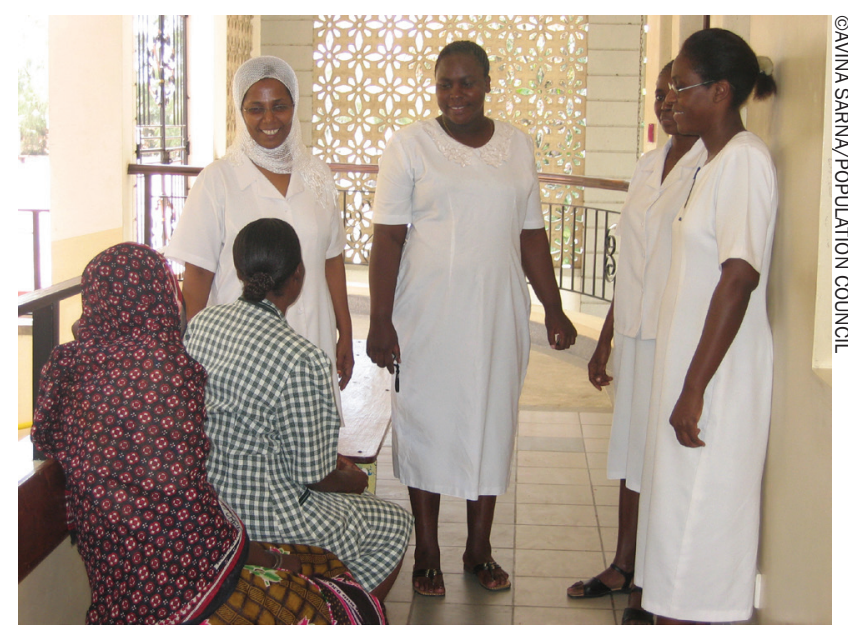

A challenge in Kenya is identifying and addressing the factors that hinder each patient's linkage to and retention in care.

promote linkage to HIV care and treatment among newly diagnosed HIV-positive clients.

This study will provide needed information to help Kenya and other countries that are scaling up test and start programs to develop effective strategies to increase uptake of HTS among men and linkage to care and treatment among all newly diagnosed HIVpositive clients.

\section{Our Research}

The first research activity is a mixed method, crosssectional study that entails (a) scoping of HTS sites in Nairobi to identify site-specific characteristics that contribute to attracting male clients for HIV testing; (b) surveying about 250 men seeking HIV testing at four high-volume HTS centers to examine their knowledge, attitudes, and practices around risk behaviors and HIV testing; and (c) undertaking qualitative in-depth interviews (IDIs) with HIVpositive men who were and were not diagnosed 
early to understand their motivations and drivers for getting tested. We are also conducting IDIs with HTS counselors to obtain provider perspectives on male clients who are diagnosed early and latetheir reasons for testing early, knowledge of HIV transmission, perceived risk and risk behaviors, persons/peers consulted about testing, expectations prior to testing, and preparedness for the results.

The second research activity consists of developing and evaluating a post-test assessment tool for HTS counselors to use to improve male and female clients' linkage to care. The activity includes a formative assessment involving in-depth interviews with health providers as well as men and women diagnosed more than six months ago who have and have not initiated antiretroviral treatment (ART). This information will inform the development of the HIV post-test assessment tool.

Project SOAR will pilot test the tool by assessing its acceptability among health providers and the feasibility of implementing it in HTS. In addition, using a two-arm quasi-experimental prospective design, the research team will assess the tool's effectiveness in linking newly diagnosed HIV-positive clients to care services and their initiation of ART. In the intervention group, counselors will be trained to use the post-test assessment tool and provide post-test counseling to clients testing positive. Counselors at comparison sites will provide routine post-test counseling as standard of care. Counselors at comparison sites will provide routine post-test counselling as standard of care.

\section{Research Utilization}

The key study stakeholders we will engage throughout the study include representatives of the Government of Kenya (specifically the National AIDS \& STI Control Program, National AIDS Control Council, and the Ministry of Health), local service providers, intervention implementing partners, the U.S. Government (United States Agency for International Development and Centers for Disease

\section{How will the post-assessment tool be used?}

The tool will comprise a checklist to guide counsellors at HTS centers to provide counselling and referrals tailored to client needs. Counsellors will be trained to use the tool to:

- Identify and focus on client characteristics that may deter the client from accessing ART services, such as lack of social support, distance from the ART center, inability to take time off from work, hopelessness or depressive symptomology, feeling healthy, etc.

- Address disclosure and stigma concerns.

- Direct clients to support services.

- Identify which clients may require further follow-up and outreach.

Control and Prevention), and others who may be identified as the study progresses. Our engagement strategies consist of holding an inception meeting with stakeholders to present and discuss the study; constituting a research advisory committee to help address study challenges, interpret research findings, and formulate recommendations; discuss and disseminate interim findings on HIV testing and linkage to care; and holding a county-level and a national-level dissemination meeting at the end of the project to inform Kenyan stakeholders of the findings and champion the recommendations.

\section{References}

${ }^{1}$ Kenya National Bureau of Statistics et al. 2015. Kenya Demographic and Health Survey 2014. Nairobi: Kenya National Bureau of Statistics. agreement funded by the President's Emergency Plan for AIDS Relief and the U. S. Agency for International Development (Agreement No. AIDOAA-A-14-00060). SOAR is able to accept funding from all USAID accounts.
Project SOAR/Population Council

4301 Connecticut Avenue, NW, Suite 280

Washington, DC 20008

Tel: +12022379400

e-mail: ProjectSOAR@popcouncil.org popcouncil.org/ProjectSOAR

(C) Population Council, December 2017 